\title{
Fx Media(Campus Social Media)
}

\author{
Robin $^{1}$ and Sahaya Jenitha ${ }^{2}$ and Santhosh ${ }^{3}$ \\ ${ }^{1}$ Department of Computer Applications, Francis Xavier Engineering College \\ ${ }^{2} \mathrm{AP}$, Department of Computer Applications, Francis Xavier Engineering \\ College
}

${ }^{3}$ Department of Computer Applications, Francis Xavier Engineering College

\begin{abstract}
In recent years, social networking system has become one of the most popular Internet applications and has a large number of users. Although the public social networking systems have meet the interpersonal communication demand of people, there is inadequate support for the closely combination of user's learning, working and cultural life on campus with their real activity. Therefore, the paper design and realized a new campus social networking system for users, which is tightly integrated with the reality environment of campus and users' real activity. It will not only achieve the basic communication function but also provide a unified platform for the teaching, researching, management and many other aspects of cultural life to teachers and students in campus. It is also an effective support for the information construction of university.
\end{abstract}

\section{KEYWORDS}

Web 2.0, BBS.

\section{INTRODUCTION}

With the wide and rapid popularity of "Face book" and "Instagram", social network (also known as social networking system or social networking service) has become one of the most popular Internet applications in recent years, and is considered to be another revolution of Internet after search engine and Web2.0 . Different with traditional BBS stations and Blog, social network no longer use anonymous way to manage users, but stressed the real-name system and establishment of network connection corresponded with the real social relationships between people. It not only emphasized the content created by users, but also highlight the spread of these content through real social relationships with the corresponding network links, in order to reduce the cost of user access to information and improve the effectiveness of information dissemination.

\section{LiTERATURE SURVEY}

\section{Social Networks Analysis, Management and Security (SNAMS-2019)}

Social network analysis is concerned with the study of relationships between social entities. The recent advances in internet technologies and social media sites, such as Facebook, Twitter and LinkedIn, have created outstanding opportunities for individuals to connect, communicate or comment on issues or events of their interests. Social networks are dynamic and evolving in nature; they also involve a huge number of users. Frequently, the information related to a certain 
concept is distributed among several servers. This brings numerous challenges to researchers, particularity in the data mining and machine learning fields. The purpose of International Conference on Social Networks Analysis, Management and Security (SNAMS-2019) is to provide a forum for researchers to present and discuss their work which is related to social network analysis

\section{Impact of Social Media on Society in a Large and Specific to Teenagers}

E-learning has been for more than around for ten years. It is probably the learning object that is assumed to be the learning content today. Commencing from computer-based delivery systems world, learning objects were idolized as Lego blocks or atoms. E-learning can be assumed as a part of lecture room or not that totally depends upon its implementation. It can be designated to as self-paced, non-synchronous training or can be drove by an instructor or can be renamed as concurrent learning. Distance learning is best matched to E-learning and also adjustable training, but can also be utilized with confronting teaching, where the terminology commonly used is blended learning. This type of blended learning in today's world is kind of static learning where the student are presented with static content irrespective of their capability to grasp the knowledge they are being provided. Therefore, there is need for a type of system that can adapt to the student's capability and would then provide knowledge by adapting to student's pace. To realize this sort of system, we would be building a system of career counselling system wherein the student would be presented a set of questions that would be based on their previous answers thus adapting to the student capability. Our system would take into consideration various factors before presenting questions to the candidate. Also, keeping in track the students previous history which also is of utmost importance, the final result that would be displayed to the student would take into consideration the historical factors of the students as well.

\section{Jinye Peng, Noboru Babaguchi, angzai Luo, Yuli Gao[2009]-Building web application in college}

Social media is a technology that can be developed in a fast and flexible manner, where internet connections are transformed into interactive platforms. Social networking programs are increasingly spreading around the world. Facebook users reached 1.44 billion per month in 2015, which means that most of the people of the world have a stamp on social networking platforms.Social media platforms have become integral part of teens'daily life. $22 \%$ of teenagers are enrolled in social networking programs about 10 times a day. The remaining category, which makes up more than half of teenagers register more than once a day through the use of mobile phone, where the proportion of teenagers who have their own cell phones reach $75 \%$. The results show by RSA that the rate of cybercrime reached $173 \%$ by mobile phones during the period 2013-2015.

\section{YasunoriShiono, TakaakiGoto, TetsuroNishino[2009]-Development of social media System}

Some areas in Asian have no medical facilities and proper mental health care is unavailable. Therefore, online counselling systems are needed. We have been studying and putting into practice online counselling for people assigned overseas. We constructed a system using agile software development for those assigned overseas in Asia. The first step involved developing a prototype system based on system requirements after we repeatedly discussed system development with people in charge of a clinic. Next, we conducted interviews about the online counselling system. We also discussed and analyzed the interviews. Finally, we completed the 
online Web counselling system by repeatedly discussing possible improvements with the clinic and then incorporating the changes in the system. We report on the construction of the system.

\section{Cui Li-xia , Liu Ya-nan, Lei Li , Tan Sheng[2010]- Relationship variables in online versus face-to-face media}

The study examined relational variables in face-to-face versus online counselling to explore how initial relationships between a counsellor and a client differed from face-to-face and online communication. The participants were 42 undergraduates who acted to come to Students Counselling center for help for the first time. Five graduates of clinical psychology acted as their counsellors. The counsellors of the two groups were same. We have been studying and putting into practice online counselling for people assigned overseas. We constructed a system using agile software development for those assigned overseas in Asia. The first step involved developing a prototype system based on system requirements after we repeatedly discussed system development with people in charge of a clinic. Social media is a technology that can be developed in a fast and flexible manner, where internet connections are transformed into interactive platforms. Social networking programs are increasingly spreading around the world. Facebook users reached 1.44 billion per month in 2015, which means that most of the people of the world have a stamp on social networking platforms.Social media platforms have become integral part of teens' daily life.

\section{Problem Statement}

\section{Architecture Diagram}

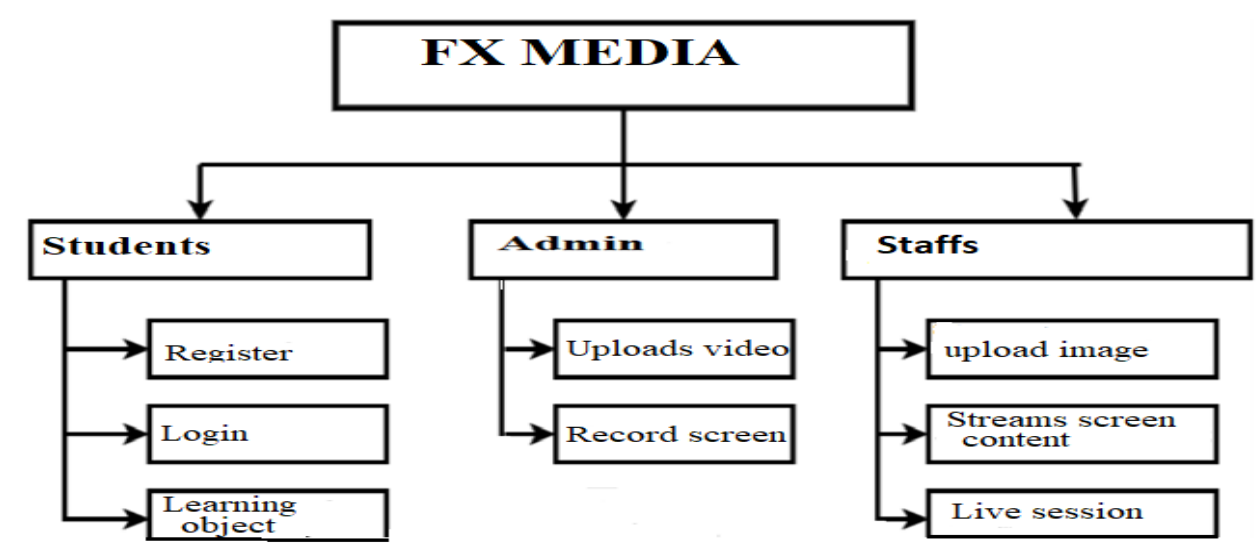

Figure 1: Architecture diagram 


\section{OUTPUt STREam}

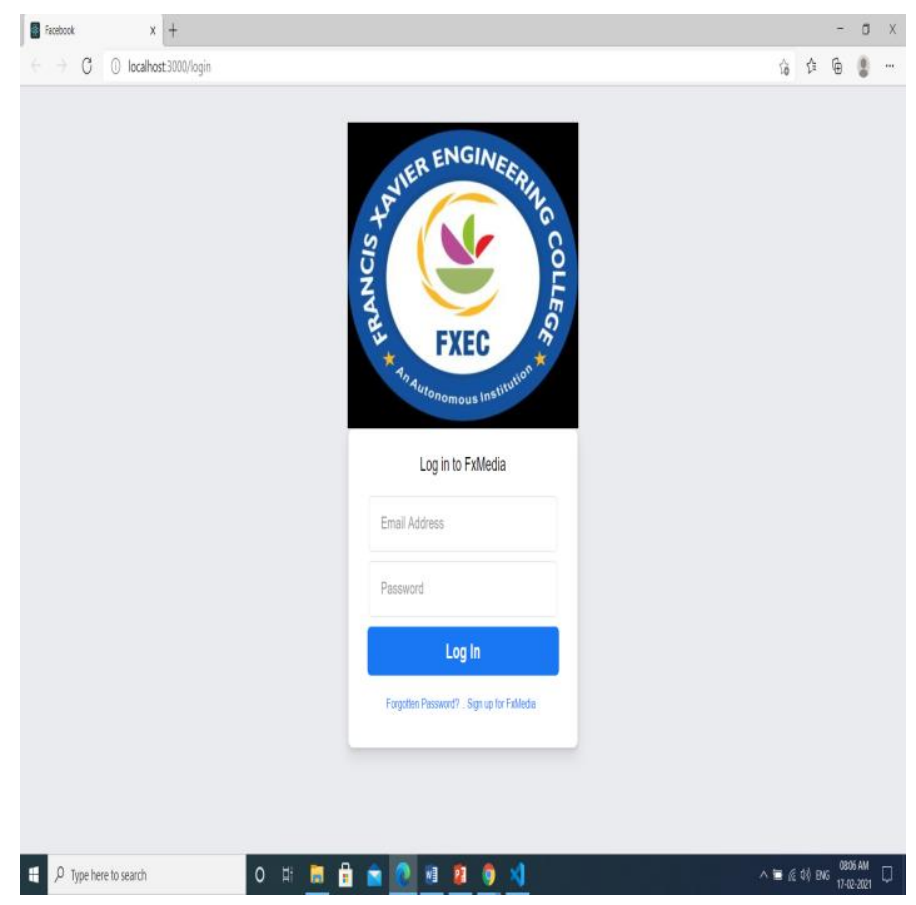

Figure 2: Login page

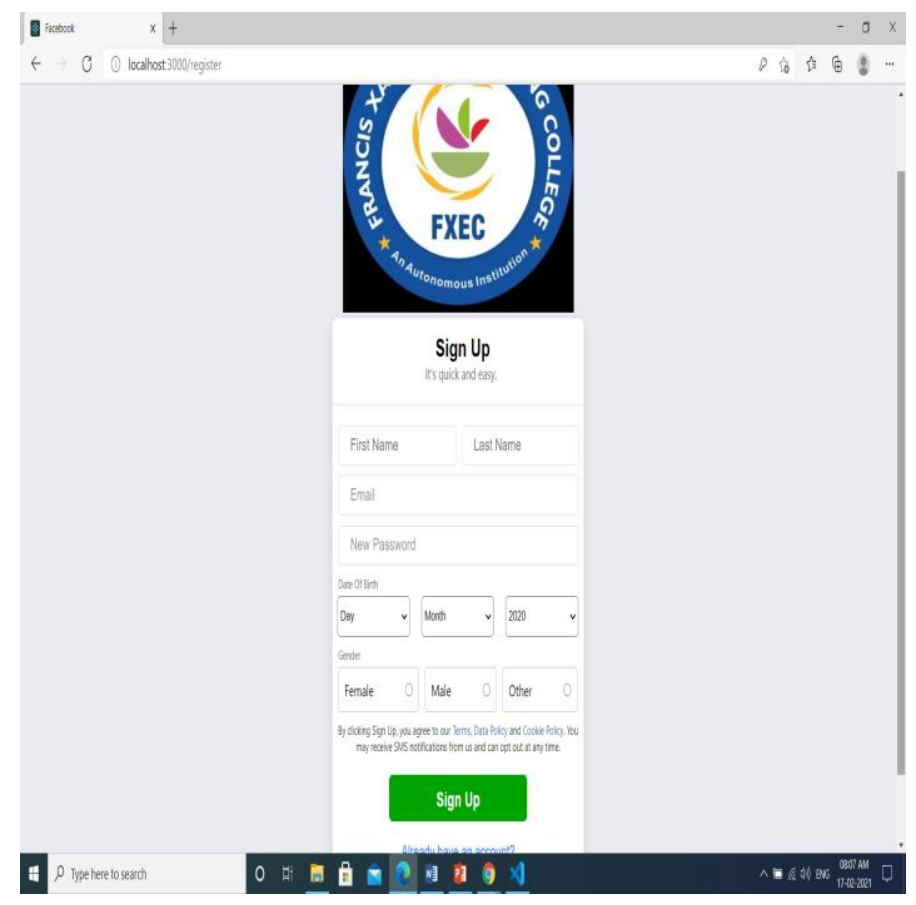

Figure 3: Sign up page 


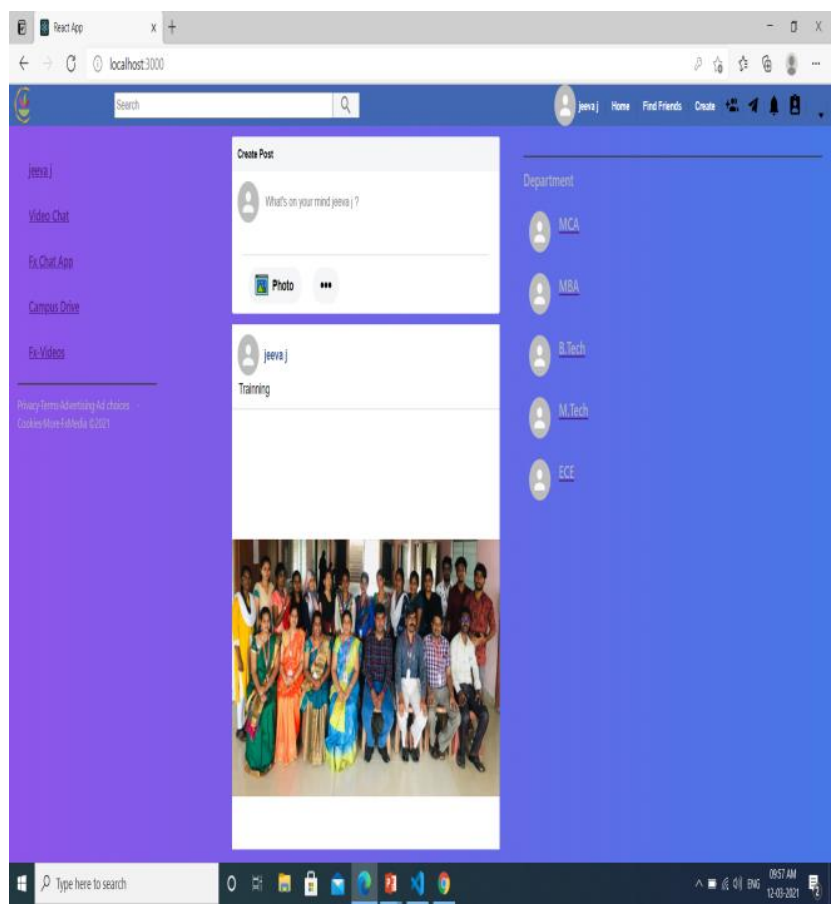

Figure 4: Home page

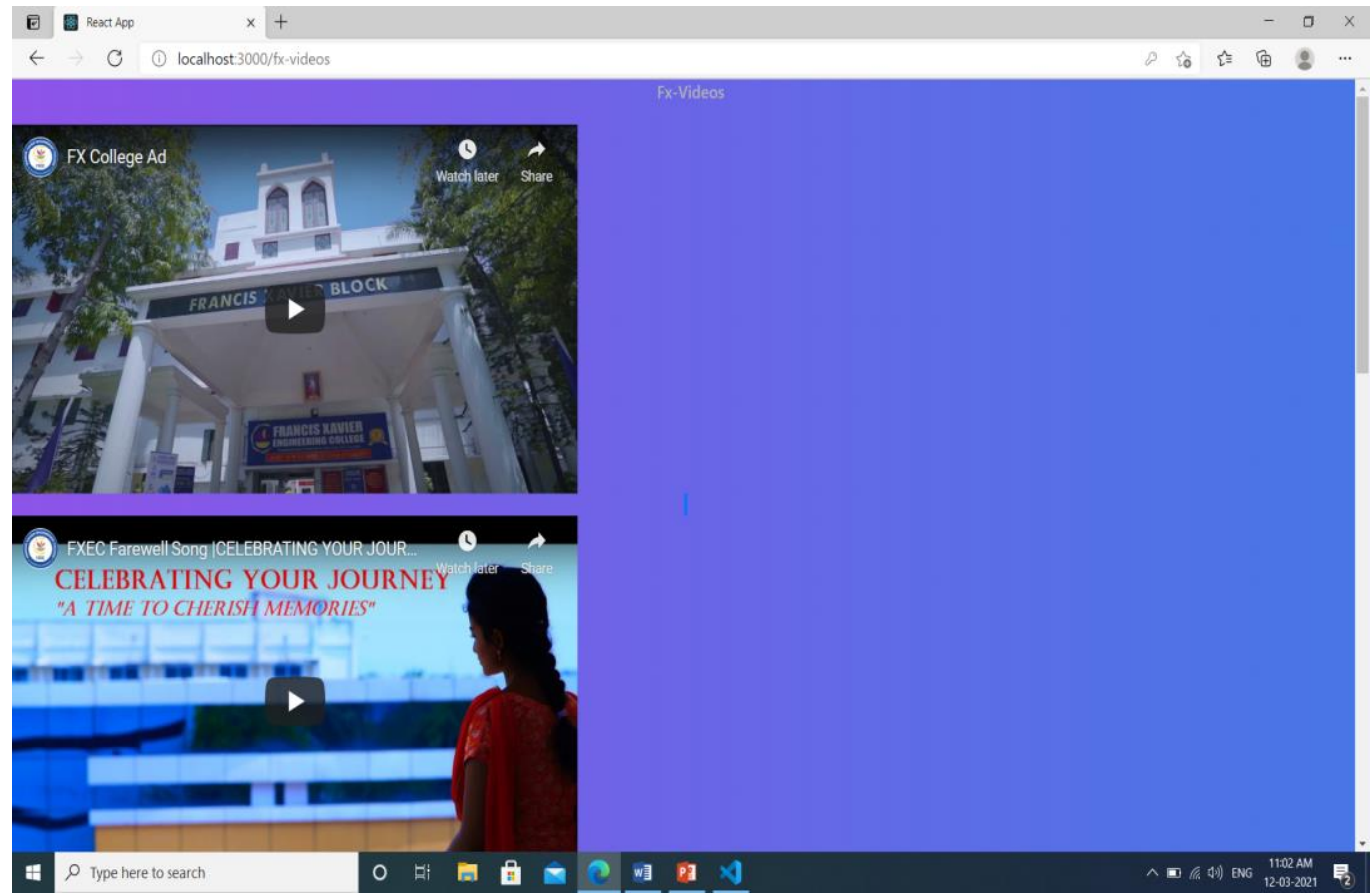

Figure 5: Ad in FX Media 


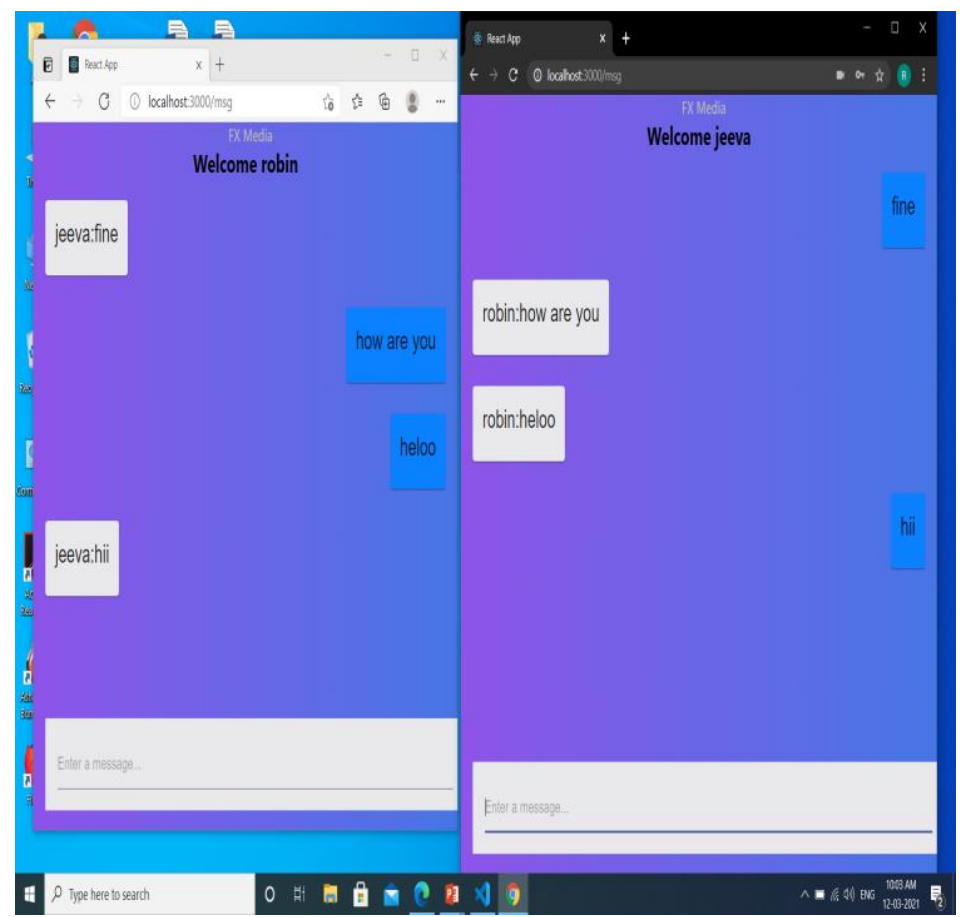

Figure 6: Chat box

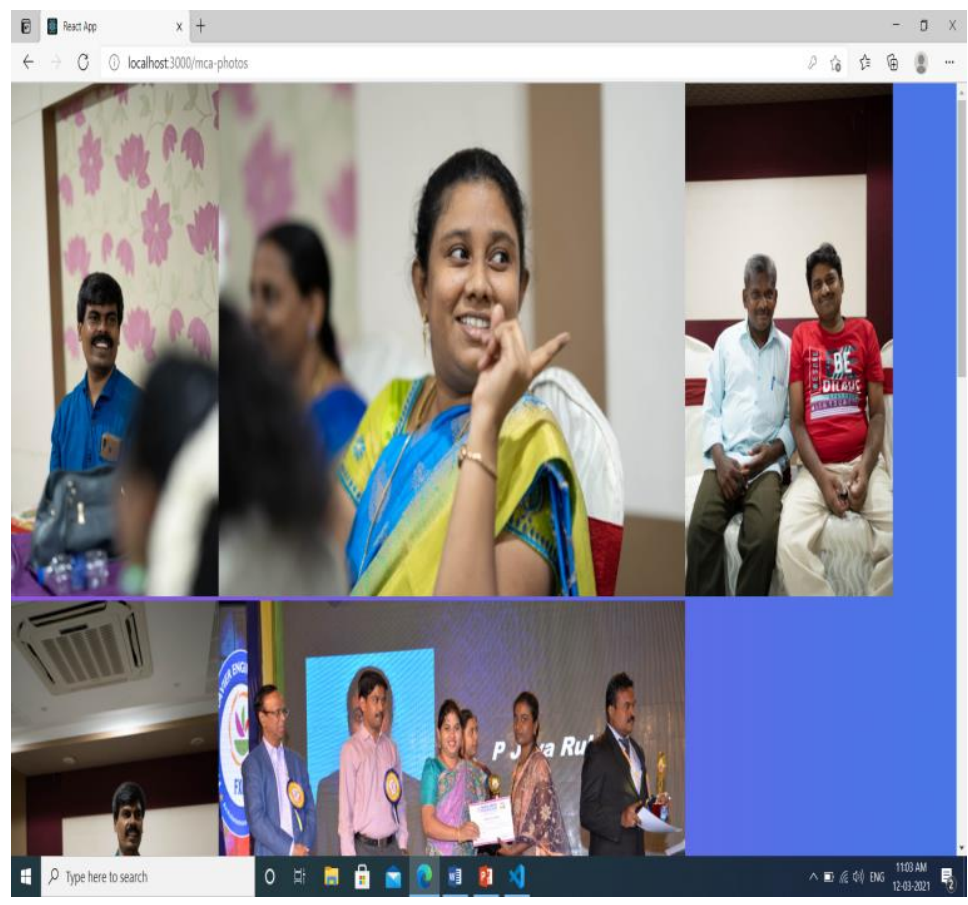

Figure 7:Gallery in FX Media 


\section{CONCLUSION}

Social networks are becoming more and more popular. More than $80 \%$ of the 4.66 billion people on the planet with access to the internet are social network users and this share is expected to grow he world is changing so fast that the urgent question is what will happen next? Will the life completely move into a virtual reality. What to expect from social media users and advertisers in 2021 and further. Today, social platforms are selling not just brand coverage, engagement, and visibility, but also very concrete indicators of direct sales.

\section{FUtURE ENHANCEMENT}

The campus social network which adopt firebase and react JS provides real time data assessment. But also provide uniform platform for the teaching researching, management and many other cultural aspects life of teachers and students in campus.

In addition it is an effective support for the information construction of university. FxMedia has offered a new platform for peer pressure with both positive and negative communication.

But in this FxMedia the negative communication has been blocked and has been enveloped by the firewall. The hole screen shared with other users.

\section{REFERENCES}

[1] [1] A Critical Analysis of Privacy and Security on Social Media -Guoying Wang,Wei Zhou,Yunsheng Zhang-2015

[2] Impact of Social Media on Society in a Large and Specific to Teenagers-Kazi Fakir Mohammed, SushoptiGawade, Vinit Nimkar- 2017

[3] Social Networks Analysis, Management and Security (SNAMS-2019)

[4] Yasunori Shiono, TakaakiGoto, TetsuroNishino[2009]- Development of campus website application

[5] Cui Li-xia, Liu Ya-nan, Lei Li , Tan Sheng[2010]- Relationship variables in online versus face-toface media. 


\section{Authors}

G. Robin doing Final Year MCA in Francis Xavier Engineering College

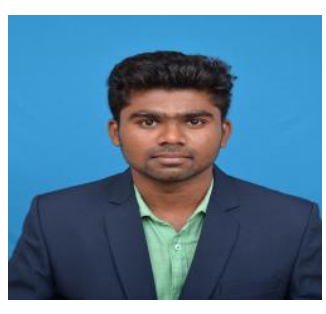

Mrs. P. Sahaya Jenitha working as Assistant Professor in the Department of Computer Applications, Francis Xavier Engineering College. Her area of interest is Wireless Sensor Networks

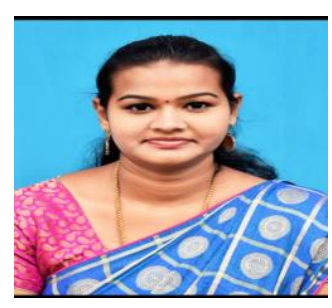

T. Santhosh doing first year MCA in Francis Xavier Engineering College

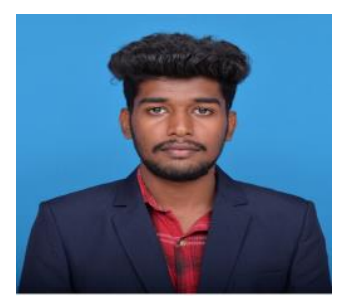

